\title{
Treatment-refractory giardiasis: challenges and solutions
}

This article was published in the following Dove Press journal: Infection and Drug Resistance

\author{
Marco Lalle' \\ Kurt Hanevik ${ }^{2,3}$ \\ 'Department of Infectious Diseases, \\ European Reference Laboratory for \\ Parasites, Istituto Superiore di Sanità, \\ Rome, Italy; ${ }^{2}$ Norwegian National \\ Advisory Unit on Tropical Infectious \\ Diseases, Department of Medicine, \\ Haukeland University Hospital, \\ Bergen, Norway; ${ }^{3}$ Department of \\ Clinical Science, Faculty of Medicine, \\ University of Bergen, Bergen, Norway
}

Correspondence: Marco Lalle Department of Infectious Diseases, Istituto Superiore di Sanità, viale Regina Elena 299, 00I6I Rome, Italy

Tel +390649902670

$\mathrm{Fax}+390649903561$

Email marco.lalle@iss.it

\begin{abstract}
Giardia is the commonest parasitic diarrheal pathogen affecting humans and a frequent cause of waterborne/foodborne parasitic diseases worldwide. Prevalence of giardiasis is higher in children, living in poor, low hygiene settings in developing countries, and in travelers returning from highly endemic areas. The clinical picture of giardiasis is heterogeneous, with high variability in severity of clinical disease. It can become chronic or be followed by post-infectious sequelae. An alarming increase in cases refractory to the conventional treatment with nitroimidazoles (ie, metronidazole) has been reported in low prevalence settings, such as European Union countries, especially in patients returning from Asia. In view of its relevance, we aim in this review to recapitulate present clinical knowledge about Giardia, with a special focus on the challenge of treatment-refractory giardiasis. We propose a working definition of clinically drug-resistant giardiasis, summarize knowledge regarding resistance mechanisms, and discuss its clinical management according to research-based evidence and medical practice. Advances in development and identification of novel drugs and potential non-pharmacological alternatives are also reviewed with the overall aim to define knowledge gaps and suggest future directions for research.
\end{abstract}

Keywords: Giardia duodenalis, giardiasis, drug resistance, treatment failure, antigiardial therapy

\section{Introduction}

Giardia duodenalis (syn. Giardia lamblia, Giardia intestinalis), hereinafter referred to as Giardia, is the etiological agent of giardiasis, one of the commonest parasitic diarrheal diseases affecting humans. ${ }^{1,2}$ This flagellated protozoan also colonizes the proximal small intestine (duodenum and jejunum) of more than 40 other mammalian species. G. duodenalis can be considered as a species complex of eight genetic groups (termed assemblages $\mathrm{A}-\mathrm{H}$ ), characterized by different host distributions and degrees of host specificity. ${ }^{3}$ Assemblages A and B are commonly associated with human infections, but also display capacity for zoonotic infection. ${ }^{3,4}$ Infection occurs by ingestion of water or food contaminated with the environmental-resistant and infectious cyst stage or by person-to-person contact or person-to-animal contact with cyst-positive feces. Following ingestion, the trophozoite stage hatches from the cyst and actively replicates and colonizes the small intestine, and the cycle completes once trophozoites differentiate into cysts and are shed in feces. ${ }^{5}$ Waterborne transmission is the main route of spread of giardiasis, but Food and Agriculture Organization of the United Nations (FAO)/WHO has also ranked Giardia as the 11th most important foodborne parasite globally. ${ }^{6,7}$ Person-to-person transmission has been documented in households infec- 
tions, and epidemic outbreaks have been documented in day care centers and institutions. ${ }^{8,9}$ The prevalence of giardiasis is highest in children younger than 5 years. Adults aged 30-40 years, returning travelers, immigrant/refugees, and homosexuals are also at risk. ${ }^{1}$ The prevalence of giardiasis is considerably higher in the developing world $(20 \%-30 \%)$ than in industrialized countries $(2 \%-5 \%) .{ }^{10}$ Poverty and poor hygienic conditions clearly promote the transmission, and the burden of giardiasis in Asia, Africa, and Latin America is still high. ${ }^{2,11}$ In industrialized countries, higher sanitary standards limit the spread of the disease, and giardiasis is perceived as a re-emerging infection being mainly and commonly associated with traveling and waterborne outbreaks., ${ }^{72}$ Nevertheless, sporadic and apparently locally acquired cases of giardiasis, not associated with travel history, have been reported and are likely to be underdiagnosed. ${ }^{10,13}$ Deprived communities and suboptimal hygienic conditions also in industrialized countries can indeed favor anthroponotic and autochthonous transmission of originally travel-acquired Giardia. ${ }^{14}$ In this review, we aim to briefly recapitulate information about clinical manifestations of giardiasis with a special focus on treatment-refractory giardiasis, in view of its relevance in countries where infection prevalence is low and exposure is relatively infrequent, such as European Union countries. A working definition of clinically drug-resistant giardiasis, the multiple mechanisms driving resistance, and the possible reasons behind its emergence are presented and discussed together with its clinical management according to research-based evidence and medical practice. Advances in development and identification of novel drugs and potential non-pharmacological alternatives are also reviewed with the final aim to define knowledge gaps and suggest future research directions.

\section{General overview on clinical manifestations of giardiasis}

The clinical picture of human giardiasis is quite heterogeneous with high variability in the severity of clinical disease. Symptoms usually appear within 2 weeks after ingestion of cysts, although asymptomatic or subclinical infection is frequent. ${ }^{1}$ Diarrhea, cramps, nausea, and vomit commonly characterize the acute phase and are often associated with fatigue and weight loss. ${ }^{1}$ Alterations and damages of the small intestinal surface, luminal enzyme deficiencies, and malabsorption may also occur. ${ }^{15}$ As the parasite is cleared, symptoms usually resolve. However, in some patients, the infection may become chronic, lasting for months or years, with or without symptoms. ${ }^{16,17}$
Both wasting and cognitive impairment have been associated with giardiasis in children, ${ }^{18}$ and early persistent Giardia infection was associated with stunting at 2 years of age as shown in the MAL-ED study. ${ }^{19}$ Giardiasis has been recognized as a significant risk factor to develop long-term post-infectious syndromes, such as post-infectious irritable bowel and chronic fatigue syndrome and extra intestinal consequences (ie, arthritis and allergies). ${ }^{20-23}$ The extreme variability in clinical manifestations of giardiasis is clearly the consequence of a complex, not yet fully elucidated, interplay between the host and the parasite. Several attempts to associate symptoms in acute giardiasis to either assemblages $\mathrm{A}$ or B were inconclusive. ${ }^{3,24}$ Chronic giardiasis is associated with host immunoglobulin deficiencies (IgA-mediated Giardia elimination is crucial at later stages of infection $)^{3}$ and malnutrition and immunosuppression ${ }^{14,15}$ Observations in animal models support a role of the host's intestinal microbiota in the susceptibility to Giardia infection and on disease pathophysiology by reciprocal interaction. ${ }^{15}$ Nevertheless, such evidence in human giardiasis is still missing.

\section{First-line antigiardial treatment options}

No effective and approved human vaccine against giardiasis is available, and pharmacotherapy is the only available option to treat giardiasis. In low-prevalence settings, treatment of confirmed cases of giardiasis is always recommended to cure symptoms and shorten the course of the disease. Moreover, effective treatment may reduce the risk of post-infectious complications and limit the spread of the infection.

A Cochrane review in 2012 identified a total of only 19 randomized controlled trials (RCTs) comparing metronidazole (MTZ) administered for 5-10 days with any of the following drugs: MTZ (single dose), tinidazole, albendazole (ABZ), mebendazole, and nitazoxanide. ${ }^{25}$ Its basic finding was that MTZ and ABZ have similar effectiveness, with ABZ having less side effects and a simpler drug regimen. There are not enough trials of other drugs to draw firm conclusions.

Effective, approved drugs consist of six classes of compounds, namely 5-nitroimidazoles (5-NIs) and benzimidazoles (BIs) derivatives, quinacrine, furazolidone, paromomycin, and nitazoxanide (Figure 1). ${ }^{26}$

NIs are prodrugs that, under intense reducing conditions in anaerobic/microaerophilic organisms, can be enzymatically reduced to highly reactive nitro or nitroso radicals that in turn can form adducts with DNA, free thiols (ie, cysteines), or protein cysteines. ${ }^{27}$ Adducts induce DNA damage, arrest of 


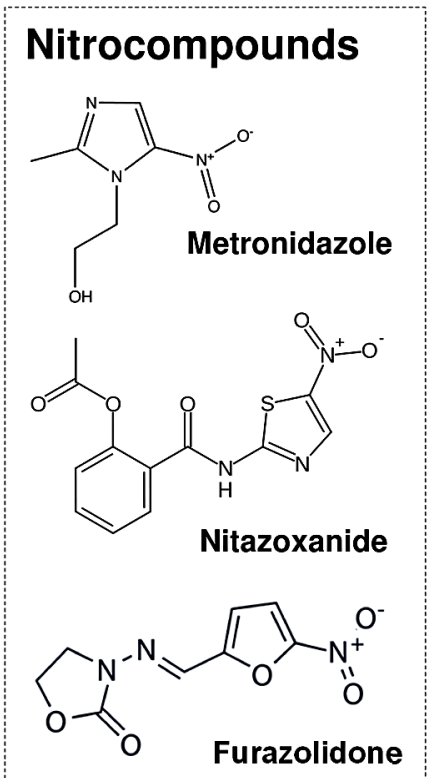

\section{Other antigiardial drugs}<smiles>CCCSc1ccc2nc(NC(=O)OC)[nH]c2c1</smiles>

Albendazole<smiles>CCN(CC)CCCC(C)Nc1ccnc2cc(Cl)ccc12</smiles>

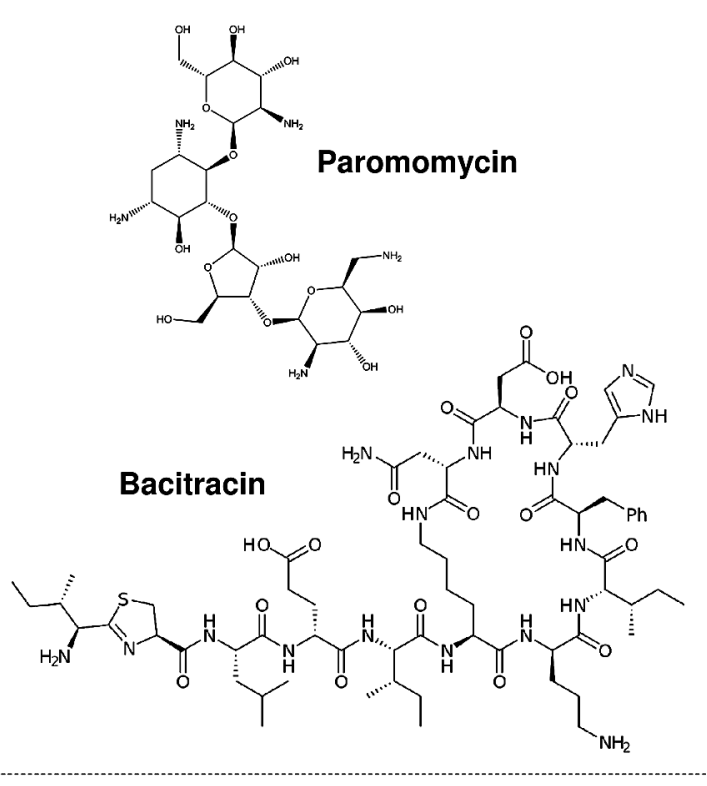

\section{New potential drugs}

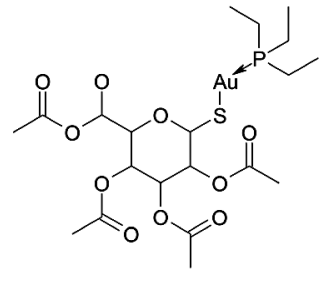

Auranofin<smiles>CO[C@H]1[C@@H](O)CC[C@@H](OC(=O)/C=C/C=C/C=C/C=C/C(=O)O)[C@H]1C1CO1</smiles>

Fumagillin<smiles>CCN(CC)C(=S)SSC(=S)N(CC)CC</smiles>

Disulfiram

Figure I Current and novel drug options for the treatment of symptomatic giardiasis.

Notes: Molecular structures of compounds correspond to those reported on PubChem (http://pubchem.ncbi.nlm.nih.gov). MTZ (PubChem number, CID 4I73), furazolidone (CID 53237I4), nitazoxanide (CID 4I684), ABZ (CID 2082), chloroquine (CID 27I9), quinacrine (CID 237), paromomycin (CID I65580), bacitracin (CID I0909430), auranofin (CID I6667669), disulfiram (CID 3II7), fumagillin (CID 69I7655), omeprazole (CID 4594), NBDHEX (CID 98I7686).

Abbreviations: $A B Z$, albendazole; MTZ, metronidazole.

the cell cycle progression, and oxidative stress that ultimately lead to parasite death. ${ }^{28}$ BIs affect the rate and the amount of microtubules assembly, and induction of oxidative stress may also play a role in the antiparasitic mechanism. ${ }^{29} \mathrm{MTZ}$ is the 5-NI prototype. It was introduced in the 1960s and listed as essential medicine by WHO. ${ }^{30}$ It is still considered the first-line antigiardial agent (cure rate of $80 \%-95 \%$ ) although unpleasant side effects are often reported. ${ }^{26}$ The common regimen is $500 \mathrm{mg}$ MTZ every 8 hours for 7-10 days (or $2 \mathrm{~g}$ for 3-5 days). Tinidazole, secnidazole, and ornidazole are used as alternatives in a single daily dose for 1 or few days, having a longer serum half-life, a higher clinical/parasitological cure rates, and milder side effects than MTZ. ${ }^{31}$ The BIs ABZ, fenbendazole, and mebendazole have both antigiardial and anthelmintic activities. Efficacy of ABZ, when administered as a single dose of $400 \mathrm{mg} /$ day for 5-10 days, is comparable to MTZ, with less reported side effects. ${ }^{25}$

\section{Treatment failure and resistance in Giardia: definition}

Regardless of the drug and the regimen used, a 100\% parasitological cure rate is rarely achieved, implying that failure in clinical treatment of some giardiasis cases is to be considered. As with bacteria, the use of antimicrobials over time ultimately leads to resistance also in parasites. In accordance with the WHO definition, drug resistance is "the ability of a parasite strain to survive and/or multiply despite the administration and absorption of a drug in doses equal to or higher than those usually recommended but within the limits of tolerance of the subject". ${ }^{32}$ Thus, drug resistance in 
Giardia is the ability of this parasite to survive in the presence of an antimicrobial drug dose that would normally kill it or limit its growth. It is customary to use the term treatment refractory when discussing clinical infections, as there may be many reasons for treatment failure, one of them being that the infecting Giardia isolate is truly drug resistant. The possibility to test or confirm drug resistance in clinical isolates is strongly hampered by the well-documented difficulty to establish routine cultures. ${ }^{33}$ Culturing, if successful, may also introduce selective bias that can alter the composition and genetic diversity in the parasite population originally present in the infected host. ${ }^{34}$ Moreover, resistance markers are not known. In view of these limitations, we propose that clinical drug-resistant giardiasis for research purposes is present when stool samples remain positive for Giardia more than 1 week after treatment completion and when other reasons for treatment failure have been excluded and the risk of reinfection is very low.

\section{Increasing prevalence of treatment- refractory giardiasis}

Treatment-refractory cases of giardiasis have been observed by clinicians for decades. However, good studies of the problem have been hampered by few and isolated treatmentrefractory cases, variable sensitivity of diagnostic methods, and the methodological issues of excluding other factors that may affect treatment success (illustrated in Figure 2). However, with improved diagnostic methods and a high patient throughput, clinically MTZ-resistant Giardia has recently been well documented in low-prevalence settings. ${ }^{35-37}$ Five decades of Giardia susceptibility to MTZ treatment is now replaced by increasing resistance, and the refractory giardiasis cases has also been reported after treatment with all other available drugs. A study of 170 giardiasis cases in Madrid from 1989 to 2004 found that 10 (5.8\%) failed one or more initial NI containing regimens; two had IgA deficiency, and one had lung cancer. ${ }^{38}$ Later, a small study in Israel reported 12 persons with NI-refractory giardiasis between 2008 and 2013. Eight of these patients who were not found to be immunodeficient were all travelers returning from Asia. ${ }^{39}$ The frequency of NI treatment-refractory cases in giardiasis patients referred to the Hospital of Tropical Diseases in London increased from $15 \%$ in 2008 to $45 \%$ in 2013. Infections originating from the Indian subcontinent were especially difficult to treat, with refractory cases reaching $70 \% .{ }^{35}$ Similarly, in a retrospective study on 95 returned Spanish travelers attending a travel clinic, 21 (22\%) had refractory giardiasis, particularly in those returning from Asian countries. ${ }^{37}$ In a more recent study involving three specialized Tropical Diseases Units in Barcelona, Spain, patients with chronic giardiasis were prospectively analyzed, and around $20 \%$ were found to be refractory to the treatment with tinidazole or MTZ. ${ }^{36}$ Also this study confirms that refractory infections originating from Asia were more prevalent $(70 \%)$ than infections originating from elsewhere. Refractory infections in the Spanish study were caused by both assemblages $\mathrm{A}$ and $\mathrm{B}$, indicating that several circulating strains have a potential for NIs resistance. Corroborating

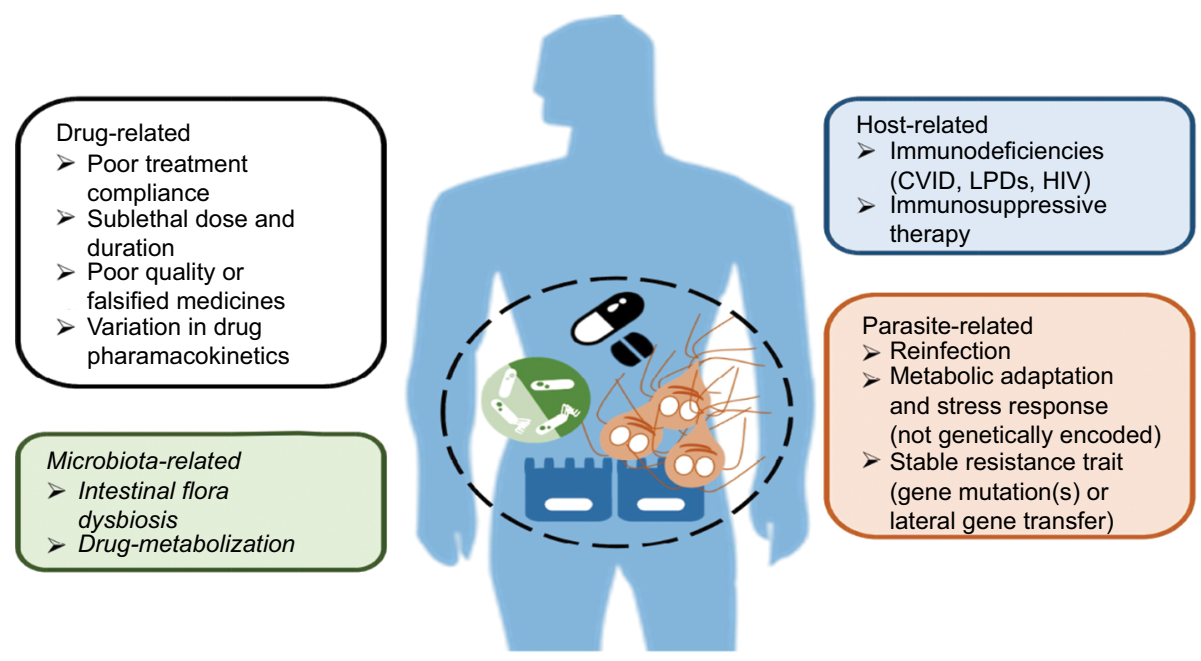

Figure 2 Overview of known and potential factors associated with clinical drug treatment failure of giardiasis.

Notes: Factors attributable to the drug, the host, the parasite, and the intestinal microbiota are listed herein and discussed in details in the main text. Factors for which evidence is still poor are written in italic.

Abbreviations: CVID, common variable immunodeficiency; LPDs, lymphoproliferative disorders. 
this, a recent Czech study genotyping 47 isolates found that nine of these (19\%) isolates originate from patients clinically resistant to MTZ. Several subgroups of assemblage AII and one assemblage BIII isolate were represented, and multidrug resistance was associated with one $\mathrm{AII}$ and one $\mathrm{AI}$ isolate. ${ }^{40}$ Interestingly, in an outbreak setting of several assemblage $B$ genotypes, ${ }^{41}$ only one genotype remained and was present in all 17 refractory isolates examined in patients referred due to treatment failure. ${ }^{42}$ This genotype likely possessed both virulence and MTZ tolerance traits that helped to establish a chronic MTZ-refractory infection that existed for several months in immunocompetent persons. Treatment failure has also been reported for ABZ either administered alone or in combination with MTZ. ${ }^{42-46}$ Studies are needed to evaluate whether this is due to a cross-resistance against $\mathrm{ABZ}$ in the now increasing MTZ-resistant isolates or is an independent resistance trait. In the regions endemic for giardiasis, intermittent administration of ABZ to children in antihelminthic programs, with a regime suboptimal for treatment of giardiasis, has indeed been associated with an increase in Giardia burdens among the patients. ${ }^{47}$

\section{Giardia treatment failure: parasite drug- resistance mechanisms}

Our knowledge about resistance mechanisms in Giardia is limited and fragmented. Drug resistance is relatively easy to induce in axenic Giardia laboratory lines, and most of the research into potential resistance mechanisms has been performed in these cell lines. Resistance can be induced through exposure of drug-susceptible trophozoites to progressively increasing concentrations of a drug over several months. ${ }^{28,48}$ Although in vitro culturing conditions cannot fully recreate the microaerophilic gut environment in vivo, resistant laboratory lines are valuable sources of knowledge about regulatory and metabolic effects of drugs and adaptations made during drug exposure.

Most studies on resistance mechanisms in Giardia have focused mainly on MTZ, but resistance mechanisms against nitazoxanide, ABZ, furazolidone, and quinacrine were also investigated, ${ }^{48}$ whereas resistance mechanisms against paromomycin, chloroquine, and bacitracin have not yet been investigated. There is also good evidence that some degree of cross-resistance is common in laboratory lines, especially between the nitro compounds. ${ }^{48,49}$ Clinical MTZ resistance has been attributed to increased MTZ tolerance in Giardia isolated from patients who failed treatment therapy. ${ }^{50,51}$ In Giardia, there are several enzymes capable of the partial reduction reaction needed to convert MTZ into toxic metabolites including the pyruvate:ferredoxin oxidoreductases (PFORs), Giardia lamblia nitroreductase 1 (GINR1), and thioredoxin reductase (TrxR). Another nitroreductase, Giardia lamblia nitroreductase 2 (GINR2), can completely deactivate MTZ by reducing it to a non-toxic aminoimidazole. A quite consistent finding has been the downregulation of GINR1 in MTZ-resistant lines, and one isolate has been shown to harbor a nonsense mutation in a third of the NRI gene transcripts, effectively reducing the levels of this protein. ${ }^{52}$ Other studies have shown less consistent results, indicating a role for PFOR, NR2 and TrxR and Flavin Mononucleotide-dependent oxidoreductases in MTZ resistance. ${ }^{53}$ Novel "omics" approaches, looking at both RNA and protein levels, have enabled studies on genes and gene products expression in isogenic MTZ-susceptible and MTZresistant laboratory lines. Several lines of evidence emphasize the broad and variable adaptive responses in resistant isotypes, including post-transcriptional and post-translational alterations. ${ }^{53-55} \mathrm{~A}$ wide range of metabolic adaptations in glycolysis, electron transport, antioxidant, and acetylation pathways have been observed in resistant laboratory lines. ${ }^{55}$ Corroborating this, other studies also attribute resistance to transcriptional plasticity and broad metabolic alterations, including decreased activity of FAD-dependent oxidoreductases, using in vitro selected MTZ- and nitazoxanide-resistant lines. ${ }^{54-56}$ It has been argued that the laboratory-induced drug resistance resembles the ability of this parasite to cope with mutable physiological conditions in the omnivorous human host,$^{56}$ which resembles the omnivorous human host where the parasite copes with mutable physiological conditions, including the increased nitrosative stress associated with an increasing meat consumption over the last century. ${ }^{57}$ Possibly, the laboratory-induced drug resistance, arising as a metabolic adaptation at many levels, should more accurately be termed "tolerance". The term "resistant" lines (or isolates, if uncloned) may be reserved for those with a stable genotypic change or acquired drug deactivating capability (ie, by lateral gene transfer), as is well documented for bacterial resistance (Figure 2). However, distinction between the two is obviously more difficult in a protozoan parasite possessing two nuclei and up to four haplotypes and generally more advanced armamentarium of regulatory capabilities than bacteria. The results obtained from laboratory-induced resistant lines should also be interpreted with some caution. The isolates from which these lines are cultured were obtained from patients several decades ago, largely belong to the zoonotic assemblage AI, and may not be representative of Giardia isolates circulating and infecting humans nowadays. ${ }^{3}$ Only a 
small fraction of Giardia isolates can be successfully grown in vitro, and there might be a strong metabolic bias in these isolates, rendering them less representative. The resistance slowly induced in laboratory lines could be of a different nature than the rapidly increasing clinical resistance seen over the last decade. The increasing resistance observed especially in travelers returning from Asia is indicative of a heritable genetic trait in Giardia.

Laboratory-induced drug resistance is generally lost or greatly reduced after removing the $\operatorname{drug}^{58}$ or passing the trophozoites through a cyst stage. ${ }^{54}$ Stability of MTZ resistance in clinical isolates from patients failing MTZ treatment is likely, as a study reported that three such isolates were also less MTZ susceptible when tested in a neonatal mouse model. ${ }^{50} \mathrm{MTZ}$ resistance comes at a virulence cost for the parasite, as some MTZ-resistant laboratory lines have either lost or greatly reduced the capacity to infect suckling mice as a consequence of mucosal attachment defect associated with an impaired glucose metabolism. ${ }^{49}$ In contrast, in other laboratory lines, induced MTZ resistance can coexist with retained infectivity. ${ }^{55}$ The laboratory line $106-2 \mathrm{ID}_{10}$ stably retained MTZ resistance after removal of MTZ for 12 weeks, showed a good growth rate, and has previously been shown to retain cytoadherence (although at lower levels) and infectivity in a suckling mouse model. ${ }^{49}$ The variation in the infectivity and molecular phenotypes of resistant Giardia lines suggests that multiple molecular resistance phenotypes are possible. Each phenotype is a complex of alterations in the genetic sequence, transcription level, and functional regulation of multiple proteins resulting in lowered susceptibility to MTZ. The finding of several assemblage subtypes in clinically MTZ-resistant cases supports this. ${ }^{40}$

\section{Giardia treatment failure: host factors and the gut microbiota}

Beside the development/selection for resistance traits in Giardia trophozoites, several reasons have been linked to clinical treatment failures including repeated infection, inappropriate drug administration, and immunological status (Figure 2). ${ }^{43}$ Reinfection is quite common in high prevalence regions where hygiene conditions are low and environmental contamination is high, so that antigiardial mass drug treatment in infected people living in these areas has been questioned. ${ }^{59}$ In low-prevalence countries, reinfection should be excluded as a primary cause of treatment failure. On the other hand, deviation from treatment compliance, such as poor adherence to the prescribed frequency and duration of medication, may occur as a consequence of drug-related bothersome side effects or difficulties with the administration of some drug formulations. ${ }^{43}$ Poor quality, counterfeit or expired drugs, and changes in drug pharmacokinetic should be excluded. Immunocompromised hosts, including patients with common variable immunodeficiency (CVID), lymphoproliferative disorders (LPDs), and HIV/ AIDS, as well as patients on immunosuppressive therapy, such as solid organ transplantation, seem to be more susceptible to giardiasis, ${ }^{60}$ and their infections are frequently more difficult to cure being often associated with treatment failure (Figure 2). ${ }^{39,43}$

Independently from the host and the parasite, a reason for ineffective treatment could be co-infection with or selection of other microorganisms in the gut microbiota. Some of these may be able to disarm the drug via metabolic inactivation (eg, by reduction of the nitro group of MTZ to the non-toxic amino derivative; Figure 2). Several microorganisms inhabiting the gut (ie, Enterococcus spp., Clostridium spp., Bacteroides spp., and Escherichia coli) may encode type I oxygen-insensitive nitroreductases that inactivate MTZ. ${ }^{21}$ A relative increase in Firmicutes (including Clostridium spp.) and Bacteroides spp., ${ }^{61}$ or an increase in Protobacteria, ${ }^{62}$ or a decrease in the amount of Lactobacillus spp in favor of Enterococcus spp. and enterobacteria $^{63}$ has been observed in several mouse models of giardiasis. Intriguingly, treatment with antibiotics ineffective against Giardia occurs in $25 \%$ of adult patients with gastrointestinal illness before receiving a correct diagnosis for giardiasis and MTZ administration. ${ }^{64}$ This interesting finding deserves further investigations. Giardia-induced dysbiosis in addition to unnecessary antibiotic treatment could potentially alter the gut flora promoting the proliferation of potentially pathogenic MTZ-resistant bacteria such as Clostridium difficile. ${ }^{21}$

\section{Management of drug treatment failure}

Treatment of giardiasis is largely based on clinical experience, and well-designed treatment trials are scarce. When the common first-line treatments with ABZ or MTZ (or tinidazole or secnidazole) monotherapy fail there are even less studies to guide treatment.

\section{Monotherapy}

In treatment-refractory giardiasis, monotherapy with a longer duration or higher dose of the same drug or treatment with new alternate drugs generally has a poorer success rate than the initial treatment for any given drug. For example, in a study using ABZ as second-line therapy in 10 NI refrac- 
tory cases, only four were cured and 1 of 4 was cured by nitazoxanide. ${ }^{39}$ Combined with other reported cases from literature, the success of ABZ monotherapy in refractory cases is below 20\%. ${ }^{39}$ Developed in the mid-1970s, nitazoxanide received its final approval by US FDA for the treatment of giardiasis in 2004. ${ }^{65-67}$ The drug is thought to interfere with the PFOR enzyme activity, which is essential for anaerobic energy metabolism. ${ }^{68}$ Nitazoxanide has few side effects, and treatment regimen is usually limited to 3 days with efficacies ranging from $71 \%$ to $85 \%$ in treatment naïve patients. ${ }^{65,66,69,70}$ An open randomized trial compared 3 days of nitazoxanide twice daily against tinidazole single dose in Cuban children and found that nitazoxanide treatment achieved parasitological cure in $78.4 \%$ of cases, compared to $90.5 \%$ with tinidazole. ${ }^{70}$ However, treatment of NI-refractory cases as a part of clinical routine practice has been reported to have lower success rates with 9 of 18 patients being cured. ${ }^{35}$

Aminoglycosides, a well-known group of antibiotics and inhibitors of protein synthesis, have been shown to be effective against Giardia. Cure rates have been relatively good when using paromomycin as a first-line treatment ${ }^{39}$ but less promising in treatment-refractory giardiasis (less than 50\%). ${ }^{35}$ Likewise, neomycin has shown positive results in a randomized clinical trial of treatment naïve individuals with 20 of 22 patients ( $86 \%$ ) being cured..$^{71}$ Furazolidone, a nitrofuran derivative, has a similar efficacy (cure rate, $80 \%-96 \%$ ) to MTZ when used as initial therapy. ${ }^{72}$ It damages the parasite through producing superoxide radicals that damage DNA and the ability to differentiate into its cyst form. ${ }^{73}$ However, aminoglycosides and furazolidone efficacy in treatment failure cases has not been investigated. The low success rate of monotherapy in treatment failure cases may be indicative of cross-resistance, as proved in vitro between MTZ and tinidazole, MTZ and ABZ, as well as between nitazoxanide and quinacrine. ${ }^{26}$

Remarkably, quinacrine (Figure 1), an old antiprotozoan compound related to chloroquine and developed in the 1930 s, is the only drug proved successful in several studies as monotherapy in treatment failure cases of giardiasis, with a near $100 \%$ cure rate. ${ }^{35-37,39}$ Quinacrine is often given as a 7 -day treatment course, ${ }^{35,37}$ although a 5-day regimen proved equally effective, even in one child for whom treatment was stopped after 3 days for adverse side effects. ${ }^{36}$ Quinacrine is generally kept as a third-line treatment option in giardiasis, mainly due to its unfavorable side effects. During a 3-week treatment course, two of three patients reported confusion, one reported dizziness, and one reported nightmares. ${ }^{42}$ Before using quinacrine, patients should always be counseled about the rare, but serious neuropsychiatric side effects, including psychosis that has been reported during the use of this drug. ${ }^{74}$ During 5 -day course of quinacrine treatment in a study on 61 treatment naïve children with giardiasis in Cuba, temporary side effects included vomiting $(23 \%)$, nausea $(23 \%)$, headache $(18 \%)$, and yellowish skin $(25 \%) .^{75}$

\section{Combination therapy}

Clinical experience of low efficacy of monotherapy has led to the use of combinations of drugs in cases of initial treatment failure. ${ }^{76}$ An early study with six treatment-refractory cases, four of which had confirmed immunodeficiency, ${ }^{43}$ found a combination of quinacrine and MTZ to be effective in five of the cases, and the sixth case finally responded to a 3-week course of quinacrine and tinidazole. Later an Italian study randomized 20 patients who had failed one to five treatment courses of MTZ to receive monotherapy with $400 \mathrm{mg} \mathrm{ABZ} \times 2$ for 7 days or combination therapy of the same $\mathrm{ABZ}$ regimen and $250 \mathrm{mg} \mathrm{MTZ} \times 3$ for 7 days. $^{77}$ While 9 of 10 responded in the combination therapy group, only 2 of 10 responded to ABZ alone. This study guided the choice of second-line treatment in 39 patients with MTZ-refractory giardiasis in Norway, where the same combination of ABZ and MTZ was successful in 79\% of patients in a treatment ladder study. Six of the patients not improving on this regimen received paromomycin that was successful in $50 \%$ of cases, and the three remaining cases were successfully treated with the combination treatment of $100 \mathrm{mg}$ quinacrine $\times 2$ and elevated dose $750 \mathrm{mg} \mathrm{MTZ}$ $\times 3$ for 2 or 3 weeks. ${ }^{42}$ In a small case series of 10 patients failing initial NI treatments, double or triple combination therapies with MTZ, ABZ, paromomycin, or quinacrine were successful in all cases. ${ }^{38}$

If drugs of the main classes fail, one may resort to drugs with a different mechanism of action. The poorly investigated drugs for giardiasis such as bacitracin zinc (Figure 1) ${ }^{71}$ may again be relevant to include in trials as monotherapy and in combinations. Chloroquine has been also used successfully in treatment of giardiasis, ${ }^{75,78}$ but no data have been published regarding its use in combination with other drugs or in NIrefractory cases.

The BI mebendazole has been shown to have efficacy against giardiasis, ${ }^{66,75}$ but it is likely to be cross-resistant with ABZ. Pyrantel pamoate, a drug licensed for use against Enterobius infection in humans, has shown a synergistic effect with the BI febantel against giardiasis in gerbils. ${ }^{76}$ The anthelmintic drug praziquantel has also been shown to have 
antigiardial effect. A number of case reports of prolonged treatments and unusual drug combinations were recently reviewed. ${ }^{76}$

\section{New assays for drug evaluation}

The implementation of high-throughput screening (HTS) assays to evaluate Giardia drug sensitivity has been accompanied by renewed efforts in the design, synthesis, and high rate identification of candidate "next-generation" antigiardial drugs. Limits associated with conventional colorimetric or fluorescent assays, due to the composition of Giardia medium, and the need to ensure microaerophilic/anaerobic conditions necessary for Giardia growth in 96-well plate format, have been successfully overcome. Microaerophilic/ anaerobic conditions can be ensured by sealing the culture plate in a bag either with anaerobic generators ${ }^{79}$ or with an airtight adhesive tape.$^{80}$ The evaluation of cellular ATP content, as index of the amount of viable cells, without removal of the Giardia culture media, has been used to screen a collection of 4,096 pharmacologically active compounds in a miniaturized 1,536-well plate format, resulting in 11 new compounds with antigiardial activity, including fumagillin, carbadox, and tioxidazole. ${ }^{79}$ The efficacy of Malaria Box, a collection of 400 diverse compounds with antimalarial activity, as well as an other 1,600 known bioactive molecules, has been tested using an automated live-cell digital phase-contrast microscopy assay that permits automated assessment of Giardia growth without cell staining. ${ }^{81,82}$ Other assays instead rely on transgenic Giardia parasites, based on either the assessment of glucuronidase activity in trophozoites ${ }^{83}$ or the amount of luciferase bioluminescence in trophozoites and encysting parasites, the latest allowing bioluminescent monitoring of the drug efficacy in mouse infection model by imaging methods. ${ }^{84}$ This last option is of paramount importance, since the increasing use of murine model of giardiasis is required for the pre-clinical evaluation of undesired pharmacokinetic/ pharmacodynamic properties.

\section{New promising anti-Giardia drugs and drug targets}

Modification of the pharmacophore is a productive strategy to improve the efficacy of existing antigiardial drugs and overcome resistance, taking also into consideration the variable drug-resistance phenotypes in this parasite. Systematic structural modifications of existing nitroheterocyclic drugs and BIs have been undertaken. ${ }^{85,86}$ As an example, more than 400 new nitroheterocyclic derivatives were obtained by modifications of the imidazole ring using click chemistry. Many of the compounds displayed better activity in vitro than MTZ and nitazoxanide, with some of them being effective $(<100$ $\mathrm{nM}$ ) against nitro drug-resistant Giardia lines. ${ }^{86}$ Interestingly, in a murine infection model, the activities of the six selected most promising compounds did not correlate well with the in vitro activities. ${ }^{86}$ This observation underlines the importance to evaluate carefully pharmacokinetic/pharmacodynamic properties when selecting compounds to further progress into optimization to clinical studies.

The creation of hybrid (chimeric) compounds, combining the features of two active molecules differing in structure and mechanisms of action, is another route for drug design. Nitazoxanide analogs, obtained by substituting the original acetylsalicylic acid moiety with different non-steroidal antiinflammatory drugs, have better antigiardial activity than MTZ and nitazoxanide, both in vitro and in a mouse model, and high selectivity index $(>50)$ vs a mammalian cell line. ${ }^{87}$

Drug repurposing of an approved drug is among the most advantageous strategies used to identify new active compounds with pre-clinical activity. With safety studies already performed, such compounds have an accelerated progression to clinical trials.

Auranofin (Figure 1) is an orally administered gold complex, rapidly metabolized to the active form of gold, and currently approved by FDA to treat rheumatoid arthritis. ${ }^{88}$ The compound is effective against Giardia in vitro and in different rodent infection models (mice and gerbils) irrespective of the assemblage used (either A or B) or the MTZ-resistance trait. ${ }^{88}$ Auranofin inhibits the recombinant TrxR in vitro, but the drug susceptibility is not increased in a Giardia line overexpressing the TrxR, ${ }^{88,89}$ indicating a mechanism of action partially independent of this enzyme. The recently solved structure of Giardia TrxR would likely help solve this issue. ${ }^{90}$ In support of future antigiardial clinical trials, the safety and good tolerability of auranofin in a short-term therapy ( $6 \mathrm{mg}$ /day orally for 7 days) has been confirmed in a phase I trial with healthy volunteers. ${ }^{91}$ Moreover, the amount of gold in feces at day 7, as a measure of the drug level, was at a concentration significantly above the in vitro $\mathrm{IC}_{50}$ for Giardia, supporting the therapeutic value of auranofin for the treatment of giardiasis. ${ }^{91}$

Fumagillin (Figure 1) is a potent antibiotic, from the fungus Aspergillus fumigatus, licensed until 2016 by the European Medicines Agency (EMEA/COMP/82/02) against microsporidian infections. It also represents the prototype of a series of angiogenesis inhibitor and anti-obesity drugs under development. ${ }^{92}$ Fumagillin targets the enzyme methionine aminopeptidase, being also effective against Plasmodium 
spp. ${ }^{93}$ Fumagillin is effective in vitro at submicromolar concentrations against both Giardia assemblage A and B and also in an adult mice infection model, using an assemblage $\mathrm{B}$ isolate, with parasite clearance at much lower doses than with MTZ. ${ }^{94}$ The capacity of fumagillin to overcome MTZ resistance in vitro ${ }^{94}$ and the observation that it can cure patients with amoebiasis and co-infection with Giardia ${ }^{95}$ make this drug promising, although the mechanism of action in Giardia still needs to be disclosed.

Disulfiram (Figure 1) is an FDA-approved drug used for years in the treatment of alcohol dependence. It prevents metabolization of acetaldehyde, the primary metabolite of alcohol, inhibiting the aldehyde dehydrogenase by dithiodiethylcarbamoyl adduct formation with a cysteine residue in the active site. Accumulation of acetaldehyde in the blood causes unpleasant effects. Disulfiram efficacy against Giardia was demonstrated in vitro with assemblage A and $\mathrm{B}$ trophozoites, in murine model of giardiasis, as well as against a MTZ-resistance isolate. ${ }^{96,97}$ Disulfiram seems to have multiple targets in Giardia as it proves to inactivate both the carbamate kinase (the terminal enzyme in the arginine dihydrolase pathway) and the Giardia lamblia triosephosphate isomerase (gITIM), a key glycolytic enzyme essential in this amitochondriate parasite, by covalent and selective thiocarbamoylation of specific cysteine residues. ${ }^{97,98}$

Omeprazole (Figure 1), lansoprazole, pantoprazole, and rabeprazole are proton pump inhibitors (PPIs) and BI derivatives approved for the treatment of acid-related diseases of the gastrointestinal tract and Helicobacter pylori infection and act as inhibitors of gastric acid secretion. PPIs accumulate as prodrugs in acidic environments and undergo acid-catalyzed conversion to the active drug. These drugs irreversibly inhibit $\mathrm{H}^{+} / \mathrm{K}^{+}$-ATPase by covalently binding to cysteine residues at the alpha-subunit. ${ }^{99}$ The short-term use of PPIs is well tolerated with few side effects. Commercially available PPIs are active in vitro against Giardia trophozoites in the range of $\mathrm{ABZ}^{100}$ and retain their activity also on MTZ-resistant strains. ${ }^{101}$ The toxic activity has been associated with the inhibition of the gITIM via the formation of covalent adducts with Cys-222 (without affecting the human enzyme homo$\log ) .{ }^{101}$ No studies using animal models of giardiasis have been conducted, but indirect evidence suggests a correlation between the use of PPIs and a reduced risk to be infected with intestinal protozoa, including Giardia. ${ }^{102}$

Exploitation of unique Giardia metabolic enzymes is another strategy undertaken for antigiardial drug discovery and development. An example is represented by the drug NBDHEX, 6-(7-nitro-2,1,3-benzoxadiazol-4-ylthio)hexanol, a promising, not approved, antitumoral compound more effective than MTZ against Giardia trophozoites in vitro (Figure 1). ${ }^{103}$ The drug inhibits the unusual FAD-dependent glycerol-3-phosphate dehydrogenase, an enzyme linked to glycolysis. A pleiotropic activity of the drug was proved later in Giardia. NBDHEX directly inhibits TrxR, forming stable covalent adducts with the catalytic Cys residues that are further reduced by TrxR to toxic nitroradicals that react with specific protein cysteines, and it likely undertakes redox cycling with the production of toxic reactive oxygen species. ${ }^{90,104}$ No data are yet available on its ability to overcome MTZ resistance or its efficacy in animal infection models.

\section{Alternative non-drug-based treatment of giardiasis}

The use of probiotics (microbial cell preparations or components of microbial cells), has also been evaluated as alternatives to, or in combination with, antigiardial pharmacotherapy, due to the low toxicity and the stimulatory effect on the host immune system. ${ }^{105}$ or Enterococcus faecium or Lactobacillus case $i^{107,108}$ to Giardia-infected gerbils, significantly reduced the amount of cysts in stools and can reduce/prevent trophozoite adhesion to the mucosal surface. ${ }^{108}$ A significant synergistic activity of $L$. case $i$ has been reported in combination with $\mathrm{ABZ}$ in a murine infection model, ${ }^{109}$ whereas in human cases of giardiasis, the administration of the probiotic yeast Saccharomyces boulardii enhanced the MTZ activity compared to MTZ monotherapy. ${ }^{110}$ The antigiardial activity of probiotic bacteria has been linked to the release of specific toxic peptides, such as bacteriocins from Lactobacillus acidophilus and Lactobacillus plantarum, ${ }^{111}$ and enzymes, such as bile salt hydrolases (BSHs) from L. johnsonii (Lal), and other lactobacilli, that produce deconjugated bile salts toxic for the parasite. ${ }^{112,113}$ The administration of either high $\mathrm{BSH}$ producer Lactobacilli or purified recombinant BSH to neonatal mouse, experimentally infected with Giardia, promotes parasite clearance. ${ }^{113}$ In addition, the administration of the iron-binding glycoprotein lactoferrin, normally present in mammalian exocrine secretions, should be also evaluated in view to the reported giardiacidal activity in vitro ${ }^{114}$ and to a lower prevalence in Giardia colonization reported in children receiving bovine lactoferrin for 9 months. ${ }^{115}$

\section{Conclusion and future perspective}

The increasing resistance toward the NIs is alarming as it prolongs the illness period and the treatment cost for giardiasis. The main challenge in treatment-refractory giardiasis is understanding of its mechanisms of resistance and 
an evidence base for its clinical management. The need to examine more recent isolates from clinical MTZ-resistant cases is of paramount importance. A heritable component is likely to be involved in the recent increase of MTZ-resistant isolates, but it may be polygenic and, at least partly, dependent on metabolic adaptation and modulation of MTZ activating/inactivating pathways. The easier access to low-cost next-generation sequencing platforms will allow whole genome sequencing and searching for hereditable resistance traits of clinical MTZ-resistant isolates. ${ }^{116}$ Methods for whole genome sequencing of cysts from stool samples enable the analysis of samples independently on their culturability. ${ }^{117}$ A systematic and integrated transcriptomic, proteomic, and even metabolomics examination should be extended beyond laboratory-induced drug-resistant lines, with the ultimate goal to define molecular tests (based on DNA, antibody, or metabolite quantification) to help clinicians making the right decisions. Since Giardia and the host microbiota influence each other and this relation might determine the course of the disease, well-designed studies to characterize (ie, by metagenomics approaches) the intestinal flora in a large cohort of patients with treatment refractory giardiasis would help define eventual microbiome signatures predictive or associated with drug failure (ie, MTZ-resistant bacteria).

In terms of treatment solutions, secondary and tertiary drug options and combinations of these are available, but there is a need for larger well-designed clinical trials of second-line drugs options against giardiasis (ie, short course of quinacrine). Although the need of brand new drugs is not yet urgent, the recently discovered compounds might secure the future of antigiardial pharmacotherapy although issues concerning their mechanisms of action, efficacy against isolates resistant to well-known drugs, and their safety and tolerability must be fully addressed to proceed into clinical trials. Probiotics, or their released peptides, in view of several promising studies, should be investigated as a potentially effective alternative or supportive therapy against drug-resistant Giardia strains or in the management of treatment-refractory cases.

\section{Acknowledgments}

Work in the laboratory of ML is supported by the Directorate-General for Health and Food Safety of the European Commission.

\section{Disclosure}

$\mathrm{KH}$ is supported by the Norwegian National Advisory Unit on Tropical Infectious Diseases, Haukeland University Hospital for his Giardia-related work. The authors report no other conflicts of interest in this work.

\section{References}

1. Cacciò SM, Lalle SM. Giardiasis. In: Xiao L, Ryan U, Feng Y, editors. Biology of Foodborne Parasites. Boca Raton: CRC Press; 2015:175-193.

2. Kirk MD, Pires SM, Black RE, et al. World Health Organization Estimates of the Global and Regional Disease Burden of 22 Foodborne Bacterial, Protozoal, and Viral Diseases, 2010: A Data Synthesis. PLoS Med. 2015;12(12):e1001921.

3. Cacciò SM, Lalle M, Svärd SG. Host specificity in the Giardia duodenalis species complex. Infect Genet Evol. Epub 2017 December 7.

4. Cacciò SM, Beck R, Lalle M, Marinculic A, Pozio E. Multilocus genotyping of Giardia duodenalis reveals striking differences between assemblages A and B. Int J Parasitol. 2008;38(13):1523-1531.

5. Adam RD. Biology of Giardia lamblia. Clin Microbiol Rev. 2001;14(3):447-475.

6. Food and Agriculture Organization of the United Nations/World Health Organization (FAO/WHO). Multicriteria-based ranking for risk management of food-borne parasites. Microbiolological Risk Assessment Series No. 23. Rome: FAO/WHO; 2014. Available from: http://www. fao.org/3/a-i3649e.pdf. Accessed May 2, 2018.

7. Efstratiou A, Ongerth JE, Karanis P. Waterborne transmission of protozoan parasites: Review of worldwide outbreaks - An update 2011-2016. Water Res. 2017;114:14-22.

8. Thompson RCA. Giardiasis as a re-emerging infectious disease and its zoonotic potential. Int J Parasitol. 2000;30(12-13):1259-1267.

9. Adam EA, Yoder JS, Gould LH, Hlavsa MC, Gargano JW. Giardiasis outbreaks in the United States, 1971-2011. Epidemiol Infect. 2016;144(13):2790-2801.

10. Hörman A, Korpela H, Sutinen J, Wedel H, Hänninen M-L. Metaanalysis in assessment of the prevalence and annual incidence of Giardia spp. and Cryptosporidium spp. infections in humans in the Nordic countries. Int J Parasitol. 2004;34(12):1337-1346.

11. World Health Organization The World Health Report 1996 [webpage on the Internet]. Fighting Disease Fostering Development. Geneva, Switzerland: World Health Organization; 1996. Available from: http:// www.who.int/whr/1996/en/. Accessed May 2, 2018.

12. Leder K, Torresi J, Libman MD, et al. GeoSentinel surveillance of illness in returned travelers, 2007-2011. Ann Intern Med. 2013;158(6):456-468.

13. Currie SL, Stephenson N, Palmer AS, Jones BL, Alexander CL. Underreporting giardiasis: time to consider the public health implications. Epidemiol Infect. 2017;145(14):3007-3011.

14. Escobedo AA, Almirall P, Hanevik K, et al. Giardiasis: a diagnosis that should be considered regardless of the setting. Epidemiol Infect. 2018;11:1-3.

15. Fink MY, Singer SM. The Intersection of Immune Responses, Microbiota, and Pathogenesis in Giardiasis. Trends Parasitol. 2017;33(11):901-913.

16. Escobedo AA, Hanevik K, Almirall P, Cimerman S, Alfonso M. Management of chronic Giardia infection. Expert Rev Anti Infect Ther. 2014;12(9):1143-1157.

17. Muhsen K, Levine MM. A systematic review and meta-analysis of the association between Giardia lamblia and endemic pediatric diarrhea in developing countries. Clinical Infectious Diseases. 2012;55(suppl_4):S271-S293.

18. Ajjampur SSR, Koshy B, Venkataramani M, et al. Effect of cryptosporidial and giardial diarrhoea on social maturity, intelligence and physical growth in children in a semi-urban slum in south India. Ann Trop Paediatr. 2011;31(3):205-212.

19. Rogawski ET, Bartelt LA, Platts-Mills JA, et al. Determinants and impact of Giardia infection in the first 2 years of life in the MAL-ED birth cohort. J Pediatric Infect Dis Soc. 2017;6(2):153-160. 
20. Litleskare S, Rortveit G, Eide GE, Hanevik K, Langeland N, Wensaas K-A. Prevalence of Irritable Bowel Syndrome and Chronic Fatigue 10 Years After Giardia Infection. Clinical Gastroenterology and Hepatology. 2018;16(7):1064-1072.

21. Halliez MCM, Buret AG. Extra-intestinal and long term consequences of Giardia duodenalis infections. World $J$ Gastroenterol. 2013;19(47):8974-8985.

22. Nakao JH, Collier SA, Gargano JW. Giardiasis and Subsequent Irritable Bowel Syndrome: A Longitudinal Cohort Study Using Health Insurance Data. J Infect Dis. 2017;215(5):798-805.

23. Dormond M, Gutierrez RL, Porter CK. Giardia lamblia infection increases risk of chronic gastrointestinal disorders. Trop Dis Travel Med Vaccines. 2016;2(1):17.

24. Robertson LJ, Hanevik K, Escobedo AA, Mørch K, Langeland N. Giardiasis - why do the symptoms sometimes never stop? Trends Parasitol. 2010;26(2):75-82.

25. Granados CE, Reveiz L, Uribe LG, Criollo CP. Drugs for treating giardiasis. Cochrane Database Syst Rev. 2012;12(5):CD007787.

26. Lalle M. Giardiasis in the post genomic era: treatment, drug resistance and novel therapeutic perspectives. Infect Disord Drug Targets. 2010;10(4):283-294.

27. Dingsdag SA, Hunter N. Metronidazole: an update on metabolism, structure-cytotoxicity and resistance mechanisms. JAntimicrob Chemother. 2018;73(2):265-279.

28. Ansell BRE, Mcconville MJ, Ma'ayeh SY, et al. Drug resistance in Giardia duodenalis. Biotechnol Adv. 2015;33(6):888-901.

29. Martínez-Espinosa R, Argüello-García R, Saavedra E, Ortega-Pierres G. Albendazole induces oxidative stress and DNA damage in the parasitic protozoan Giardia duodenalis. Front Microbiol. 2015;6(286):800.

30. World Health Organization [webpage on the Internet]. WHO Model List of Essential Medicines (20th List). 2017. Available from: http://www. who.int/medicines/publications/essentialmedicines/en/. Accessed May 2, 2018.

31. Ordóñez-Mena JM, Mccarthy ND, Fanshawe TR. Comparative efficacy of drugs for treating giardiasis: a systematic update of the literature and network meta-analysis of randomized clinical trials. J Antimicrob Chemother. 2018;73(3):596-606.

32. Basco L, Ringwald P. Drug-resistant malaria: problems with its definition and technical approaches. Sante. 2000;10(1):47-50.

33. Isaac-Renton JL, Shahriari H, Bowie WR. Comparison of an in vitro method and an in vivo method of Giardia excystation. Appl Environ Microbiol. 1992;58(5):1530-1533.

34. Andrews RH, Chilton NB, Mayrhofer G. Selection of specific genotypes of giardia intestinalis by growth in vitro and in vivo. Parasitology. 1992;105(03):375-386.

35. Nabarro LEB, Lever RA, Armstrong M, Chiodini PL. Increased incidence of nitroimidazole-refractory giardiasis at the Hospital for Tropical Diseases, London: 2008-2013. Clinical Microbiology and Infection. 2015;21(8):791-796.

36. Requena-Méndez A, Goñi P, Rubio E, et al. The Use of Quinacrine in Nitroimidazole-resistant Giardia Duodenalis: An Old Drug for an Emerging Problem. J Infect Dis. 2017;215(6):946-953.

37. Muñoz Gutiérrez J, Aldasoro E, Requena A, et al. Refractory giardiasis in Spanish travellers. Travel Med Infect Dis. 2013;11(2):126-129.

38. Lopez-Velez R, Jiménez C, Perez-Molina J, Batlle C, Navarro M, Norman F. Short course combination therapy for giardiasis after nitroimidazole failure. Am J Trop Med Hyg. 2010;83(1):171-173.

39. Meltzer E, Lachish T, Schwartz E. Treatment of Giardiasis after Nonresponse to Nitroimidazole. Emerg Infect Dis. 2014;20(10):1738-1740

40. Lecová L, Weisz F, Tůmová $\mathrm{P}$, Tolarová V, Nohýnková E. The first multilocus genotype analysis of Giardia intestinalis in humans in the Czech Republic. Parasitology. 2018;820:11-1111. Epub 2018 Mar 20.

41. Robertson LJ, Forberg T, Hermansen L, Gjerde BK, Langeland N. Molecular characterisation of Giardia isolates from clinical infections following a waterborne outbreak. J Infect. 2007;55(1):79-88.
42. Mørch K, Hanevik K, Robertson LJ, Strand EA, Langeland N. Treatment-ladder and genetic characterisation of parasites in refractory giardiasis after an outbreak in Norway. J Infect. 2008;56(4):268-273.

43. Nash TE, Ohl CA, Thomas E, Subramanian G, Keiser P, Moore TA. Treatment of Patients with Refractory Giardiasis. Clinical Infectious Diseases. 2001;33(1):22-28.

44. Kollaritsch H, Jeschko E, Wiedermann G. Albendazole is highly effective against cutaneous larva migrans but not against Giardia infection: results of an open pilot trial in travellers returning from the tropics. Trans R Soc Trop Med Hyg. 1993;87(6):689.

45. Brasseur P, Favennec L. Two cases of giardiasis unsuccessfully treated by albendazole. Parasite. 1995;2(4):422.

46. Abboud P, Lemée V, Gargala G, et al. Successful Treatment of Metronidazole- and Albendazole-Resistant Giardiasis with Nitazoxanide in a Patient with Acquired Immunodeficiency Syndrome. Clinical Infectious Diseases. 2001;32(12):1792-1794.

47. Quihui-Cota L, Morales-Figueroa GG. Persistence of intestinal parasitic infections during the national de-worming campaign in schoolchildren of northwestern Mexico: a cross-sectional study. Ann Gastroenterol. 2012;25(1):57-60.

48. Leitsch D. Drug Resistance in the Microaerophilic Parasite Giardia lamblia. Curr Trop Med Rep. 2015;2(3):128-135.

49. Tejman-Yarden N, Millman M, Lauwaet T, et al. Impaired parasite attachment as fitness cost of metronidazole resistance in Giardia lamblia. Antimicrob Agents Chemother. 2011;55(10):4643-4651.

50. Lemée Vet al. Metronidazole and albendazole susceptibility of 11 clinical isolates of Giardia duodenalis from France. J Antimicrob Chemother. 2000;46(5):819-821.

51. Adagu IS, Nolder D, Warhurst DC, Rossignol JF. In vitro activity of nitazoxanide and related compounds against isolates of Giardia intestinalis, Entamoeba histolytica and Trichomonas vaginalis. JAntimicrob Chemother. 2002;49(1):103-111.

52. Ansell BRE, Baker L, Emery SJ, et al. Transcriptomics indicates active and passive metronidazole resistance mechanisms in three seminal Giardia lines. Front Microbiol. 2017;8(Pt 4):398.

53. Leitsch D. A review on metronidazole: an old warhorse in antimicrobial chemotherapy. Parasitology. 2017;6:1-12. Epub 2017 Nov 23.

54. Müller J, Ley S, Felger I, Hemphill A, Müller N. Identification of differentially expressed genes in a Giardia lamblia WB C6 clone resistant to nitazoxanide and metronidazole. JAntimicrob Chemother. 2008;62(1):72-82.

55. Emery SJ, Baker L, Ansell BRE, et al. Differential protein expression and post-translational modifications in metronidazole-resistant Giardia duodenalis. Gigascience. 2018;7(4):Accessed May 14, 2018. https:// academic.oup.com/gigascience/article-abstract/7/4/giy024/4931738.

56. Müller J, Hemphill A, Müller N. Physiological aspects of nitro drug resistance in Giardia lamblia. Int J Parasitol. 2018;8(2):271-277.

57. Hughes R, Cross AJ, Pollock JR, Bingham S. Dose-dependent effect of dietary meat on endogenous colonic N-nitrosation. Carcinogenesis. 2001;22(1):199-202.

58. Smith NC, Bryant C, Boreham PFL. Possible roles for pyruvate: Ferredoxin oxidoreductase and thiol-dependent peroxidase and reductase activities in resistance to nitroheterocyclic drugs in Giardia intestinalis. Int J Parasitol. 1988;18(7):991-997.

59. Saffar MJ, Qaffari J, Khalilian AR, Kosarian M. Rapid reinfection by Giardia lamblia after treatment in a hyperendemic area: the case against treatment. East Mediterr Health J. 2005;11(1-2):73-78.

60. Solaymani-Mohammadi S, Singer SM. Giardia duodenalis: The double-edged sword of immune responses in giardiasis. Exp Parasitol. 2010;126(3):292-297.

61. Beatty JK, Akierman SV, Motta J-P, et al. Giardia duodenalis induces pathogenic dysbiosis of human intestinal microbiota biofilms. Int $J$ Parasitol. 2017;47(6):311-326.

62. Barash NR, Maloney JG, Singer SM, Dawson SC. Giardia Alters Commensal Microbial Diversity throughout the Murine Gut. Infect Immun. 2017;85(6):e00948-16. 
63. Pavanelli MF, Colli CM, Gomes ML, et al. Comparative study of effects of assemblages AII and BIV of Giardia duodenalis on mucosa and microbiota of the small intestine in mice. Biomed Pharmacother. 2018;101:563-571.

64. Beer KD, Collier SA, du F, Gargano JW. Giardiasis Diagnosis and Treatment Practices Among Commercially Insured Persons in the United States. Clinical Infectious Diseases. 2017;64(9):1244-1250.

65. Rossignol JF, Ayoub A, Ayers MS. Treatment of Diarrhea Caused by Giardia intestinalis and Entamoeba histolytica or E. dispar: A Randomized, Double-Blind, Placebo-Controlled Study of Nitazoxanide. J Infect Dis. 2001;184(3):381-384.

66. Rodríguez-García R, Rodríguez-Guzmán LM, Cruz del Castillo AH. Effectiveness and safety of mebendazole compared to nitazoxanide in the treatment of Giardia lamblia in children. Rev Gastroenterol Mex. 1999;64(3):122-126.

67. Fox LM, Saravolatz LD. Nitazoxanide: A New Thiazolide Antiparasitic Agent. Clinical Infectious Diseases. 2005;40(8):1173-1180.

68. Hoffman PS, Sisson G, Croxen MA, et al. Antiparasitic drug nitazoxanide inhibits the pyruvate oxidoreductases of Helicobacter pylori, selected anaerobic bacteria and parasites, and Campylobacter jejuni. Antimicrob Agents Chemother. 2007;51(3):868-876.

69. Ortiz JJ, Ayoub A, Gargala G, Chegne NL, Favennec L. Randomized clinical study of nitazoxanide compared to metronidazole in the treatment of symptomatic giardiasis in children from Northern Peru. Alimentary Pharmacology and Therapeutics. 2001;15(9):1409-1415.

70. Escobedo AA, Alvarez G, González ME, et al. The treatment of giardiasis in children: single-dose tinidazole compared with 3 days of nitazoxanide. Annals of Tropical Medicine \& Parasitology. 2008;102(3):199-207.

71. Andrews BJ, Vasile-Bugarin AC, Vasiliu RP, Jipa GH, Panitescu D, Ronnevig JR. Chemotherapy for giardiasis: randomized clinical trial of bacitracin, bacitracin zinc, and a combination of bacitracin zinc with neomycin. Am J Trop Med Hyg. 1995;52(4):318-321.

72. Quiros-Buelna E. Furazolidone and Metronidazole for Treatment of Giardiasis in Children. Scand J Gastroenterol. 1989;24(sup169):65-69.

73. Hausen MA, Freitas JCM, Monteiro-Leal LH. The effects of metronidazole and furazolidone during Giardia differentiation into cysts. Exp Parasitol. 2006;113(3):135-141.

74. Genel F, Erermis S, Aksu G, Ozturk C, Kutukculer N. Quinacrineinduced psychiatric disturbances in a child with common variable immunodeficiency and chronic giardiasis. Hum Psychopharmacol. 2002;17(7):357-359.

75. Cañete R, Escobedo AA, Gonzalez ME, Almirall P. Randomized clinical study of five days apostrophe therapy with mebendazole compared to quinacrine in the treatment of symptomatic giardiasis in children. World J Gastroenterol. 2006;12(39):6366-6370.

76. Escobedo AA, Lalle M, Hrastnik NI, et al. Combination therapy in the management of giardiasis: What laboratory and clinical studies tell us, so far. Acta Trop. 2016;162:196-205.

77. Cacopardo B, Patamia I, Bonaccorso V, di Paola O, Bonforte S, Brancati G. Synergic effect of albendazole plus metronidazole association in the treatment of metronidazole-resistant giardiasis. Clin Ter. 1995;146(12):761-767.

78. Escobedo AA, Núñez FA, Moreira I, Vega E, Pareja A, Almirall P. Comparison of chloroquine, albendazole and tinidazole in the treatment of children with giardiasis. Annals of Tropical Medicine \& Parasitology. 2003;97(4):367-371.

79. Chen CZ, Kulakova L, Southall N, et al. High-Throughput Giardia lamblia Viability Assay Using Bioluminescent ATP Content Measurements. Antimicrob Agents Chemother. 2011;55(2):667-675.

80. Bénéré E, da Luz RAI, Vermeersch M, Cos P, Maes L. A new quantitative in vitro microculture method for Giardia duodenalis trophozoites. J Microbiol Methods. 2007;71(2):101-106.

81. Gut J, Ang KKH, Legac J, Arkin MR, Rosenthal PJ, Mckerrow JH. An image-based assay for high throughput screening of Giardia lamblia. J Microbiol Methods. 2011;84(3):398-405.

82. Hart CJS, Munro T, Andrews KT, et al. A novel invitro imagebased assay identifies new drug leads for giardiasis. Int J Parasitol. 2017;7(1):83-89.
83. Müller J, Nillius D, Hehl A, Hemphill A, Müller N. Stable expression of Escherichia coli -glucuronidase A (GusA) in Giardia lamblia: application to high-throughput drug susceptibility testing. JAntimicrob Chemother. 2009;64(6):1187-1191.

84. Barash NR, Nosala C, Pham JK, et al. Giardia Colonizes and Encysts in High-Density Foci in the Murine Small Intestine. mSphere. 2017;2(3):e00343-16.

85. Flores-Carrillo P, Velázquez-López JM, Aguayo-Ortiz R, et al. Synthesis, antiprotozoal activity, and chemoinformatic analysis of 2-(methylthio)-1 H -benzimidazole-5-carboxamide derivatives: Identification of new selective giardicidal and trichomonicidal compounds. Eur J Med Chem. 2017;137:211-220.

86. Kim WJ, Korthals KA, Li S, et al. Click Chemistry-Facilitated Structural Diversification of Nitrothiazoles, Nitrofurans, and Nitropyrroles Enhances Antimicrobial Activity against Giardia lamblia. Antimicrob Agents Chemother. 2017;61(6):e02397-16.

87. Colín-Lozano B, León-Rivera I, Chan-Bacab MJ, et al. Synthesis, in vitro and in vivo giardicidal activity of nitrothiazole-NSAID chimeras displaying broad antiprotozoal spectrum. Bioorg Med Chem Lett. 2017;27(15):3490-3494.

88. Tejman-Yarden N, Miyamoto Y, Leitsch D, et al. A reprofiled drug, auranofin, is effective against metronidazole-resistant Giardia lamblia. Antimicrob Agents Chemother. 2013;57(5):2029-2035.

89. Leitsch D, Müller J, Müller N. Evaluation of Giardia lamblia thioredoxin reductase as drug activating enzyme and as drug target. Int J Parasitol. 2016;6(3):148-153.

90. Brogi S, Fiorillo A, Chemi G, et al. Structural characterization of Giardia duodenalis thioredoxin reductase (gTrxR) and computational analysis of its interaction with NBDHEX. Eur J Med Chem. 2017;135:479-490.

91. Capparelli EV, Bricker-Ford R, Rogers MJ, Mckerrow JH, Reed SL. Phase I Clinical Trial Results of Auranofin, a Novel Antiparasitic Agent. Antimicrob Agents Chemother. 2016;61(1):e01947-16.

92. Howland RH. Aspergillus, Angiogenesis, and Obesity: The Story Behind Beloranib. J Psychosoc Nurs Ment Health Serv. 2015;53(3):13-16.

93. Kang J-M, Ju J-W, Kim J-Y, et al. Expression and biochemical characterization of a type I methionine aminopeptidase of Plasmodium vivax. Protein Expr Purif. 2015;108:48-53.

94. Kulakova L, Galkin A, Chen CZ, et al. Discovery of Novel Antigiardiasis Drug Candidates. Antimicrob Agents Chemother. 2014;58(12):7303-7311.

95. Killough JH, Magill GB, Smith RC. The Treatment of Amebiasis with Fumagillin. Science. 1952;115(2977):71-72.

96. Nash T, Rice WG. Efficacies of zinc-finger-active drugs against Giardia lamblia. Antimicrob Agents Chemother. 1998;42(6):1488-1492.

97. Galkin A, Kulakova L, Lim K, et al. Structural Basis for Inactivation of Giardia lamblia Carbamate Kinase by Disulfiram. Journal of Biological Chemistry. 2014;289(15):10502-10509.

98. Castillo-Villanueva A, Rufino-González Y, Méndez S-T, et al. Disulfiram as a novel inactivator of Giardia lamblia triosephosphate isomerase with antigiardial potential. Int J Parasitol. 2017;7(3):425-432.

99. Nehra AK, Alexander JA, Loftus CG, Nehra V. Proton Pump Inhibitors: Review of Emerging Concerns. Mayo Clin Proc. 2018;93(2):240-246.

100. Pérez-Villanueva J, Romo-Mancillas A, Hernández-Campos A, YépezMulia L, Hernández-Luis F, Castillo R. Antiprotozoal activity of proton-pump inhibitors. Bioorg Med Chem Lett. 2011;21(24):7351-7354.

101. Reyes-Vivas H, de La Mora-de La Mora I, Castillo-Villanueva A, et al. Giardial Triosephosphate Isomerase as Possible Target of the Cytotoxic Effect of Omeprazole in Giardia lamblia. Antimicrob Agents Chemother. 2014;58(12):7072-7082.

102. Sheele JM. Proton Pump Inhibitor Use Is Associated With a Reduced Risk of Infection with Intestinal Protozoa. Wilderness Environ Med. 2017;28(4):339-341.

103. Lalle M, Camerini S, Cecchetti S, et al. The FAD-dependent glycerol3-phosphate dehydrogenase of Giardia duodenalis: an unconventional enzyme that interacts with the g14-3-3 and it is a target of the antitumoral compound NBDHEX. Front Microbiol. 2015;06(51):544.

104. Camerini S, Bocedi A, Cecchetti S, et al. Proteomic and functional analyses reveal pleiotropic action of the anti-tumoral compound NBDHEX in Giardia duodenalis. Int J Parasitol. 2017;7(2):147-158. 
105. Vitetta L, Saltzman ET, Nikov T, Ibrahim I, Hall S. Modulating the Gut Micro-Environment in the Treatment of Intestinal Parasites. J Clin Med. 2016;5(11):102.

106. Humen MA, de Antoni GL, Benyacoub J, et al. Lactobacillus johnsonii La1 antagonizes Giardia intestinalis in vivo. Infect Immun. 2005;73(2):1265-1269.

107. Benyacoub J, Pérez PF, Rochat F, et al. Enterococcus faecium SF68 enhances the immune response to Giardia intestinalis in mice. J Nutr. 2005;135(5):1171-1176.

108. Shukla G, Devi P, Sehgal R. Effect of Lactobacillus casei as a probiotic on modulation of giardiasis. Dig Dis Sci. 2008;53(10):2671-2679.

109. Shukla G, Kaur H, Sharma L. Comparative therapeutic effect of probiotic Lactobacillus casei alone and in conjunction with antiprotozoal drugs in murine giardiasis. Parasitol Res. 2013;112(6):2143-2149.

110. Besirbellioglu BA, Ulcay A, Can M, et al. Saccharomyces boulardii and infection due to Giardia lamblia. Scand J Infect Dis. 2006;38(6-7):479-481.

111. Amer EI, Mossallam SF, Mahrous H. Therapeutic enhancement of newly derived bacteriocins against Giardia lamblia. Exp Parasitol. 2014;146:52-63.
112. Travers MA, Sow C, Zirah S, et al. Deconjugated Bile Salts Produced by Extracellular Bile-Salt Hydrolase-Like Activities from the Probiotic Lactobacillus johnsonii La1 Inhibit Giardia duodenalis In vitro Growth. Front Microbiol. 2016;7:1453.

113. Allain T, Chaouch S, Thomas M, et al. Bile Salt Hydrolase Activities: A Novel Target to Screen Anti-Giardia Lactobacilli? Front Microbiol. 2018;9:89.

114. Turchany JM, Aley SB, Gillin FD. Giardicidal activity of lactoferrin and N-terminal peptides. Infect Immun. 1995;63(11):4550-4552.

115. Ochoa TJ, Chea-Woo E, Campos M, et al. Impact of lactoferrin supplementation on growth and prevalence of Giardia colonization in children. Clin Infect Dis. 2008;46(12):1881-1883.

116. Aurrecoechea C, Brestelli J, Brunk BP, et al. GiardiaDB and TrichDB: integrated genomic resources for the eukaryotic protist pathogens Giardia lamblia and Trichomonas vaginalis. Nucleic Acids Res. 2009;37(Database issue):D526-D530.

117. Hanevik K, Bakken R, Brattbakk HR, Saghaug CS, Langeland N. Whole genome sequencing of clinical isolates of Giardia lamblia. Clin Microbiol Infect. 2015;21(2):192.e1-3.
Infection and Drug Resistance

\section{Publish your work in this journal}

Infection and Drug Resistance is an international, peer-reviewed openaccess journal that focuses on the optimal treatment of infection (bacterial, fungal and viral) and the development and institution of preventive strategies to minimize the development and spread of resistance. The journal is specifically concerned with the epidemiology of antibiotic

\section{Dovepress}

resistance and the mechanisms of resistance development and diffusion in both hospitals and the community. The manuscript management system is completely online and includes a very quick and fair peerreview system, which is all easy to use. Visit http://www.dovepress.com testimonials.php to read real quotes from published authors. 